\title{
GEORGES-HENRI SOUTOU
}

\section{La diplomatie française et les diplomates français entre tradition et réforme}

Identité nationale, américanisation, européanisation, mondialisation: cette problématique est bien sûr au cœur de la diplomatie française depuis 1945. Celle-ci a été marquée évidemment par des évolutions et des adaptations, mais aussi par certaines continuités, en particulier le souci de maintenir l'identité nationale: la France a toujours été le moins atlantiste des pays d'Europe occidentale et le plus rétif au leadership américain; ses élites se sont en général méfiées de la mondialisation libérale, souhaitant, de Pierre Mendès France à François Mitterrand, promouvoir telle ou telle forme de »libéralisme organisé« limitant le jeu du marché, en particulier pour les matières premières, ainsi que pour les productions culturelles (»exception culturelle« et francophonie). Quant à l'européanisation, elle a constitué certainement un axe de la diplomatie et des diplomates français, mais elle est restée partielle et chargée d'arrière-pensées très »nationales«. De façon peut-être excessive, mais pas fausse, les partenaires de la France au sein de la CEE puis de l'Union européenne avaient (ont toujours) l'impression que Paris considère l'Europe avant tout comme un levier permettant de prolonger son action et de diffuser son modèle; de Gaulle lui-même ne parlait-il pas de l'Europe comme d'un »levier d'Archimède« pour la France? C'est au fond ce que signifie le qualificatif de "Grande Nation« que les Allemands appliquent volontiers à la France, en y mêlant, dans des proportions variables, ironie et admiration...

\section{5-1947 \\ UNE TENTATIVE DE RESTAURATION D’UNE POLITIQUE NATIONALE TRADITIONNELLE}

En 1945, la France, pensant pouvoir retrouver son rôle international traditionnel, commença par renouer avec l'organisation, les méthodes et les conceptions de la diplomatie d'avant-guerre. La seule véritable nouveauté résida dans la formation des futurs diplomates, qui désormais passaient par l'ENA et non plus par le concours spécifique des Affaires étrangères ${ }^{1}$. Cependant la formation qu'ils y recevaient renforçait sans doute leur connaissance de la France, dans le cadre d'une fonction publique dorénavant

1 Georges-Henri Soutou, Les élites diplomatiques françaises et allemandes au $\mathrm{XX}^{\mathrm{e}}$ siècle, dans: Rainer HudemanN, Georges-Henri Soutou (dir.), Eliten in Deutschland und Frankreich im 19. und 20. Jahrhundert/Elites en France et en Allemagne aux $\mathrm{XIX}^{\mathrm{e}}$ et $\mathrm{XX}^{\mathrm{e}}$ siècles, Munich 1994, p. 303-314. 
unifiée, mais paradoxalement diminuait plutôt leur connaissance du monde extérieur. Cela dit, le système typiquement français des grandes écoles et des grands corps techniques de l'État assurait et assure la formation d'un milieu administratif dirigeant homogène, avec sans doute moins de distance qu'ailleurs en Occident entre les diplomates d'une part et les responsables, en particulier, des finances publiques, de l'économie et du commerce extérieur de l'autre, ce qui est bien sûr essentiel pour les phénomènes envisagés $\mathrm{ici}^{2}$. Citons d'autres exemples: les contacts plus étroits qu'avant-guerre entre militaires et diplomates, en particulier dans le cadre du secrétariat général de la Défense nationale, même si cet organisme n'a jamais eu la même importance que le National Security Council aux États-Unis. Citons également le fait que le secrétaire général du Quai d'Orsay est membre de droit du Comité de l'énergie atomique, où est discutée la politique nucléaire (militaire et civile) et où de très nombreuses décisions ont été prises concernant la France, mais aussi les rapports avec l'Europe, l'Amérique et le tiers monde ${ }^{3}$. Malgré ses défauts du point de vue de la formation professionnelle des diplomates (défaut qu'après 1997 M. Hubert Védrine, alors ministre des Affaires étrangères, voulut corriger par la création au Quai d'Orsay de l'Institut diplomatique, chargé de la formation permanente des jeunes diplomates), il est certain que la création de l'ENA et les réorganisations administratives d'après 1945 et sous la $\mathrm{V}^{\mathrm{e}}$ République permirent une modernisation du corps diplomatique, le rendant plus apte à traiter d'affaires qui n'étaient plus essentiellement politiques et juridiques mais de plus en plus économiques et techniques (au sens des grands systèmes techniques pour l'énergie et les communications) et aussi politico-stratégiques.

Cependant, on n'en était pas encore là aux lendemains de la guerre. Les transformations du monde à l'issue du conflit n'étaient encore que partiellement prises en compte. Certes, on avait conscience de l'importance de l'Asie pour la suite du $\mathrm{XX}^{\mathrm{e}}$ siècle, ce fut l'une des raisons de la volonté de rester présent en Indochine. L'Empire, rénové sous la forme de l'Union française, devrait étayer ce rôle mondial ${ }^{4}$. Certes, la France participait à la nouvelle ONU, mais une partie seulement des élites dirigeantes y voyait véritablement un axe essentiel de la politique extérieure française, et à San Francisco l'un des soucis essentiels de la délégation française fut que la charte n'empêchât pas la conclusion d'alliances bilatérales (avec l'URSS et la Grande-Bretagne) de type traditionnel, réagissant justement contre la »sécurité collective« de l'entre-deux-guerres, considérée désormais avec suspicion ${ }^{5}$. Quant à la politique à l'égard de l'Allemagne vaincue, elle renouait explicitement avec les objectifs de 1914-1924 (contrôle écono-

2 La carrière d'Olivier Wormser, directeur des affaires économiques au Quai d'Orsay, plus tard ambassadeur à Moscou puis gouverneur de la Banque de France est un excellent exemple de ces synergies. On attend la thèse de Laurent WARLOUZET, université de Paris IV.

3 Georges-Henri Soutou, La logique d'un choix: le CEA et le problème des filières électronucléaires, 1953-1969, Relations Internationales 68 (1991), p. 351-377; ID., Pierre Guillaumat, le CEA et le nucléaire civil, dans: Georges-Henri Soutou, Alain BeLTRAN (dir.), La passion des grands projets industriels (Institut d'histoire de l'industrie), Paris 1995, p. 97-130.

4 Pierre GERBET, Le relèvement 1944-1949, Paris 1991.

5 Georges-Henri SouTOU, La France et la création de l'ONU, 1944-1946, à paraître. 
mique et géopolitique de la Sarre, de la Rhénanie, de la Ruhr) ${ }^{6}$. Les diplomates les plus importants de cette période (Dejean, Massigli, Chauvel) pouvaient diverger sur bien des points, mais restaient tous dans un cadre intellectuel national classique, et se méfiaient même désormais de la sécurité collective, que certains d'entre eux, comme Massigli, avaient pourtant soutenue dans les années $1930^{7}$.

Le seul domaine novateur était celui de l'économie internationale, devant l'évidence de l'échec dramatique de la politique protectionniste suivie durant les années 1930: dès 1945 Paris se ralliait, au moins en principe, aux objectifs anglo-saxons d'un monde ouvert et d'un marché mondial restauré, appuyé sur un système monétaire international stabilisé. Jean Monnet, au Commissariat au plan, portait cette orientation nouvelle et y ajoutait avant même la fin de la guerre une dimension européenne; au Quai, Hervé Alphand, directeur des affaires économiques, suivait ces orientations, plaidait pour l'abandon du protectionnisme et pour la prise en compte des besoins de l'économie européenne globale dans le règlement de l'affaire allemande, se distinguant par là fortement des objectifs géopolitiques beaucoup plus traditionnels des dirigeants politiques, du général de Gaulle à Georges Bidault (au moins jusqu'en 1947) ${ }^{8}$.

\section{$1948-1958$ \\ L'EMPIRE, L'EUROPE ET L'ATLANTIQUE}

Jusqu'en 1958 l'Union française, recentrée après la perte de l'Indochine et avec la guerre d'Algérie autour du concept d'Eurafrique, resta au cœur de la vision française du monde ${ }^{9}$. Mais l'adaptation de cette vision aux nouvelles réalités mondiales (opposition de l'URSS et des États-Unis aux empires traditionnels, apparition du tiers monde, conférence de Bandoeng) ne se fit pas tout de suite, ou mal, comme le démontre l'affaire de Suez en $1956^{10}$. Notons cependant ici les profondes divisions qui existèrent à Paris au sujet de cette crise. Le Quai d'Orsay était majoritairement (pas unanimement) hostile au recours à la force et à la collusion avec Israël, estimant que les positions traditionnelles de la France au Moyen-Orient en seraient compromises, sans

6 Georges-Henri Soutou, La politique française à l'égard de la Rhénanie 1944-1947, dans: Peter HÜTTENBERGER, Hangsgeorg MOLITOR (dir.), Franzosen und Deutsche am Rhein 1789 1918 - 1945, Essen, 1989, p. 47-66; Georges-Henri SoutoU, Frankreich und die Deutschlandfrage 1943 bis 1945, dans: Hans-Erich VolKMANN (dir.), Ende des Dritten Reiches - Ende des Zweiten Weltkrieges, Munich 1995, p. 54-75.

7 Maurice Dejean n'a pas laissé de mémoires; cf. ses papiers aux archives du ministère des Affaires étrangères et Georges-Henri SouTOU, Le général de Gaulle et l'URSS, 1943-1945: idéologie ou équilibre européen, dans: Revue d'histoire diplomatique (1994) 4, p. 303-356; René MASsigLI, Une comédie des erreurs, Paris 1978; Raphaële ULRICH-PIER, René Massigli (1888-1988). Une vie de diplomate, Bruxelles 2006; Jean Chauvel, Commentaire, 3 vol., Paris 1973.

8 SOUTOU, Frankreich und die Deutschlandfrage (voir n. 6); Jean MonNET, Mémoires, Paris 1976.

9 Charles-Robert AGERON, La décolonisation française, Paris 1994.

10 Maurice VAÏSSE (dir.), La France et l'opération de Suez de 1956, Paris 1997. 
bénéfice appréciable pour la guerre d'Algérie. Il fut d'ailleurs tenu en dehors de la préparation de l'expédition ${ }^{11}$. L'armée de terre au contraire estimait indispensable d'abattre Nasser pour interrompre le soutien de l'Égypte à la rébellion algérienne. Mais le président du Conseil, Guy Mollet, ainsi que son ministre des Affaires étrangères, Christian Pineau, et le ministère de la Défense, avec à sa tête Maurice BourgèsMaunoury, avaient une vision beaucoup plus large, dépassant l'Algérie, dont ils pensaient de toute façon que l'indépendance (dans le cadre de telle ou telle forme d'association à la France) serait à terme inéluctable: il fallait soutenir Israël (auquel les rattachaient les souvenirs de la Résistance et des options socialistes convergentes) afin d'empêcher le nationalisme arabe, appuyé sur l'URSS, de contrôler le Moyen-Orient, avec ses voies stratégiques et son pétrole. Notons que le Quai d'Orsay et ses adversaires dans cette affaire tirèrent de la crise des leçons très différentes: le ministère des Affaires étrangères estimait que la France avait durablement compromis ses positions dans le monde arabe, sans aucun bénéfice pour la guerre d'Algérie, et que les grands vainqueurs étaient finalement Nasser, dont le prestige sortait renforcé de la crise, et l'URSS, dont la pénétration au Moyen-Orient serait facilitée ${ }^{12}$. Au ministère de la Défense, on faisait encore en 1957 une analyse toute différente et beaucoup plus optimiste: Israël, allié essentiel, avait été sauvé, la progression de Nasser avait été bloquée, un coup d'arrêt avait été donné à la pénétration soviétique au Moyen-Orient, la »doctrine Eisenhower« pour cette région, proclamée en janvier 1957, témoignait que finalement Washington se ralliait aux vues des Français et ne cherchait plus à appuyer le nationalisme nassérien comme une garantie contre la poussée soviétique ${ }^{13}$.

On peut noter qu'avec sa vision »traditionnelle« du monde arabe, le Quai d'Orsay se trompait finalement moins dans ses analyses que les »modernistes« autour de BourgèsMaunoury, désireux de défendre les intérêts français dans le contexte nouveau de la guerre froide, du pétrole-roi et des armes nucléaires (qui figuraient très haut dans les priorités de cette équipe, qui négocia avec Bonn et Rome pour la mise en commun des efforts atomiques des trois pays, là aussi contre l'avis du Quai d'Orsay, et qui décida également de collaborer avec Israël dans ce domaine) ${ }^{14}$. Plus qu'une opposition en fait sans réelle signification entre »traditionalistes« et »modernistes«, ce sont en fait des visions différentes qui sont en cause, mais portant sur des problèmes très actuels: le Quai comprend que la politique américaine ne se résume pas à un atlantisme systématique et n'est pas surpris du >lâchage « des États-Unis dans une affaire dont ils n'avaient d'ailleurs pas été tenus réellement informés; quant à l'Europe, contre l'enthousiasme simpliste de certains milieux, on comprend que l'on ne peut pas faire n'importe quoi en son nom dans un domaine aussi sensible que le nucléaire, sous peine de provoquer

11 Cf. le récit de Bernard Destremeau, Quai d'Orsay derrière la façade, Paris 1994, p. 155-176.

12 Note de la Direction politique du 10 novembre 1956, Documents Diplomatiques Français 1956/III.

13 Communication à paraître.

14 Georges-Henri SouTOU, L'Alliance incertaine. Les rapports politico-stratégiques francoallemands, 1954-1996, Paris 1996; Pierre PÉAN, Histoire de deux bombes, Paris 1991. 
de vives réactions à Washington et à Moscou; et enfin, à propos du tiers monde, on comprend que l'ère des interventions militaires européennes est close.

Cependant, le début de la guerre froide et l'échec des projets initiaux à l'égard de l'Allemagne, la mise en place du plan Marshall d'abord, puis de l'Alliance atlantique, et les débuts de la construction européenne suscitèrent un développement de la diplomatie multilatérale, un début de réorganisation du Quai d'Orsay et un changement de paradigmes: désormais on admettait que le maintien du rôle international de la France nécessitait des relais, européens ou atlantiques, pouvant aller pour certains jusqu'à l'intégration. Les diplomates français prirent l'habitude d'une concertation permanente avec leurs collègues britanniques et américains, dans le cadre de très nombreuses réunions tripartites à différents niveaux ainsi que dans le travail régulier des ambassades situées dans des pays-clés pour la guerre froide (Moscou et Belgrade constituant de bons exemples) ${ }^{15}$. Cette concertation était facilitée par le développement au Quai d'Orsay même de nouveaux services voués à la diplomatie multilatérale: en particulier la Sous-direction de la coopération économique internationale, avec Olivier Wormser, qui géra en particulier la participation française au plan Marshall et à l'OECE et par la suite au marché commun ${ }^{16}$, et le Service des pactes, qui suivait les questions de 1'Alliance atlantique et les affaires stratégiques ${ }^{17}$. Quant au secrétariat général, outre son rôle traditionnel de gestion de la maison, il suivait directement les grandes questions multilatérales liées à la guerre froide: Allemagne, OTAN, URSS, communisme international ${ }^{18}$.

Ce développement du multilatéralisme débouchait même chez certains diplomates (au premier rang Hervé Alphand, directeur général des affaires économiques et négociateur de la CED) jusqu'aux concepts de l'intégration et de la supranationalité, à la suite de Jean Monnet et de Robert Schuman et conformément aux principes d'organisation de l'OTAN, et encore plus de la CECA et de la CED ${ }^{19}$. Incontestablement, à travers la coopération étroite avec les États-Unis et l'Alliance atlantique, à travers les nombreuses réunions d'experts, une certaine américanisation se faisait jour, car il ne faut pas sous-estimer l'influence pédagogique modernisatrice de cette coopération, ainsi qu'une incontestable européanisation ${ }^{20}$. Le Quai d'Orsay sortait des années 1930.

15 Cf. par exemple Georges-Henri SouTou, La politique française envers la Yougoslavie, 19451956, dans: Relations internationales 104 (2000), p. 433-454.

16 Précisons: la Direction des affaires économiques et financières, hostile au départ au marché commun, avait été largement écartée des négociations menant aux traités de Rome au profit du Secrétariat général à la coopération internationale (SGCI) mais elle revint au premier plan en 1958 quand on décida de lui rattacher la représentation de la France à Bruxelles et à constituer une structure duale DAEF-SGCI; Laurent WARLOUZET, Le Quai d'Orsay face au traité de Rome, dans: Laurence BADEL, Stanislas JEANNESSON, N. Piers LudLOW (dir.), Les administrations nationales et la construction européenne, Berne 2005, p. 141.

17 Pour l'activité du Service des pactes cf. par exemple SouTOU, L'Alliance incertaine (voir n. 14).

18 Georges-Henri Soutou, La perception du problème soviétique par le Quai d'Orsay entre 1945 et 1949, dans: Revue d'Allemagne (1998) 3, p. 273-284.

19 Hervé ALPhand, L'étonnement d'être, Paris 1977.

20 On trouvera des indications sur ce point dans Henri Froment-Meurice, Vu du Quai. Mémoires 1945-1983, Paris 1998. 
En même temps, ces tendances étaient combattues à l'intérieur de la Maison, elles restaient sans doute minoritaires, même si la discipline des fonctionnaires estompait ce fait $^{21}$. En particulier la France fut toujours le moins atlantiste des pays européens, et cela valait également pour son corps diplomatique. La majorité des diplomates réconciliaient les évidentes nécessités atlantiques et européennes d'une part, et l'identité nationale d'autre part, en considérant que la collaboration atlantique permettait à la France de conserver son statut mondial à l'instar de la Grande-Bretagne, et que la construction européenne était désormais le seul moyen possible pour la France de conserver un certain ascendant sur l'Allemagne et un contrôle sur l'évolution de la question allemande ${ }^{22}$. Les diplomates vraiment »atlantistes« ou »européens « et qui ne partageaient pas ces motivations très »nationales« étaient certainement minoritaires. Certaines arrière-pensées de réassurance discrète à Moscou, en particulier afin de contrer un rapprochement germano-américain considéré comme excessif, montrent bien la permanence d'une certaine vision géopolitique traditionnelle ${ }^{23}$. Dès 19531954, il était clair que les diplomates français étaient loin de tous se rallier aux nouveaux paradigmes européens et atlantiques ${ }^{24}$. Les mouvements de personnel sous Mendès France, en particulier l'arrivée au secrétariat général de René Massigli, montraient certes la volonté de tourner la page après les déchirements provoqués au sein du Quai par la querelle de la CED, mais aussi le recul de l'idée de l'intégration européenne ${ }^{25}$. En 1955, on vit encore chez certains diplomates la force de l'atlantisme, c'est-àdire de la conviction que face à Moscou l'unité occidentale était la priorité ${ }^{6}$. Mais la crise algérienne et celle de Suez devaient, dès 1956, réactiver une volonté d'indépendance nationale toujours très présente chez beaucoup de membres du Quai d'Orsay ${ }^{27}$. Dès cette année, l'intégration, qu'elle fût atlantique ou européenne, était condamnée aux yeux de beaucoup d'entre eux, et dans la préparation des traités de Rome, le Quai d'Orsay veilla à ce que l'intégration supranationale fût beaucoup moins prononcée que dans la cas de la CECA ou de la CED. Cela ne signifiait pas que l'on n'était pas convaincu de la nécessité de collaborer avec les États-Unis et les Européens, mais selon des méthodes relevant davantage de la coopération interétatique

21 Cf. les remarques de DeStremeaU, Quai d'Orsay (voir n. 11), p. 117-121.

22 Georges-Henri Soutou, La perception de la menace soviétique par les décideurs de l'Europe occidentale: le cas de la France, dans: Saki DockRILL, Robert FRANK, Georges-Henri SouTOU, Antonio VARSORI (dir.), L'Europe de l'Est et de l'Ouest dans la Guerre froide 1948-1953, Paris 2002, p. 21-43.

23 Georges-Henri Soutou, La France et les notes soviétiques de 1952 sur l'Allemagne, Revue d'Allemagne juil.-sep. (1988), p. 261-273.

24 Georges-Henri SoutoU, Georges Bidault et la construction européenne 1944-1954, dans: Serge Berstein, Jean-Marie MAYEur, Pierre Milza (dir.), Le MRP et la construction européenne, Bruxelles 1993, p. 197-230.

25 Cf. Soutou, Les élites diplomatiques (voir n. 1).

26 Georges-Henri SouTOU, Les Français et la question d'une éventuelle politique soviétique de »neutralité« en Europe, 1954-1955, communication à paraître, et ID., Unter der Lupe: Frankreich und Adenauers Reise nach Moskau, communication à paraître.

27 Jacques BAEYENS, Un coup d'épée dans l'eau du canal, Paris 1976. 
traditionnelle que de l'intégration et de la supranationalité. Cela annonçait en fait la politique extérieure gaulliste, dont beaucoup d'éléments d'ailleurs se mettaient en place dans les esprits avant même la fin de la IV ${ }^{\mathrm{e}}$ République.

\section{8-1990 \\ INDÉPENDANCE NATIONALE ET NOUVEAU CONCERT EUROPÉEN}

De nombreux diplomates, sans doute la majorité, furent satisfaits en effet de revenir à la politique plus traditionnelle d'indépendance nationale qui marqua la $\mathrm{V}^{\mathrm{e}}$ République $^{28}$. Ajoutons qu'ils furent sans aucun doute soulagés de sortir des incertitudes et contradictions de la $\mathrm{IV}^{\mathrm{e}}$ République, y compris en matière de politique extérieure, et que la politique gaulliste avait le mérite de la clarté et de la cohérence. Cela n'empêchait pas une pratique habile des institutions bruxelloises, les représentants permanents de la France auprès des communautés réalisant au fond la synthèse entre la diplomatie multilatérale et la construction européenne d'une part, et la défense de l'intérêt national de l'autre. En effet, le général ne contestait pas les institutions de Bruxelles, les soutenait même, à condition qu'elles ne sortent pas de leur rôle, ne se prennent pas pour un super-État en gestation, et que leur action contribue à développer ce qu'il appelait une $»$ Europe européenne ${ }^{29}$. Fondamentalement, la distance prise par rapport à l'Alliance atlantique et la pratique plus musclée de l'Europe bruxelloise, ainsi que le projet de nouveau Concert européen qui était au cœur du gaullisme, et le rôle en flèche de la France durant ces années, suscita une large adhésion et par la suite une nostalgie sensible jusqu'à maintenant. Le »grand dessein « gaulliste consistait en effet à recréer un équilibre européen modernisé reposant sur une série d'équilibres croisés (la France conservant son ascendant sur la RFA grâce à l'appui discret d'une Russie de moins en moins idéologisée, l'Europe occidentale sous leadership français permettant en revanche de rééquilibrer l'URSS). Cette »géopolitique à la française« reprenait au fond une tradition vivante depuis le $\mathrm{XIX}^{\mathrm{e}}$ siècle, tandis que les prises de position du général de Gaulle face à la »double hégémonie« et contre les blocs flattaient l'amourpropre national, même si elles paraissaient parfois excessives à des diplomates qui auraient préféré un langage plus modéré; mais dès le début des années 1960 les diplomates vraiment »européens« ou »atlantistes«, réservés à l'égard de la politique gaulliste, furent placés sur des voies de garage (certes honorables: dans cette première phase, la $\mathrm{V}^{\mathrm{e}}$ République ne pratiquait pas »la mise au placard «) ${ }^{30}$.

28 Maurice Couve DE Murville, Une politique étrangère 1958-1969, Paris 1971; François SEYDOUX, Dans l'intimité franco-allemande, Paris 1977.

29 Alain PrATE, Les batailles économiques du général de Gaulle, Paris 1978.

30 Pour les orientations fondamentales du gaullisme telles que je les interprète ici, cf. SOUTOU, L'Alliance incertaine (voir n. 14) et ID., Le général de Gaulle, le plan Fouchet et l'Europe, dans: Commentaire 52 (1990/1991), p. 757-766. 
Quant au projet d'une Europe politique reposant non pas sur l'intégration mais sur la coopération interétatique et assurant le leadership français, sans compromettre l'indépendance nationale, grâce à la division de l'Allemagne et à l'absence de la GrandeBretagne, elle séduisit de nombreux diplomates (même si certains persistaient à considérer l'Angleterre comme un partenaire privilégié plus souhaitable que la RFA). C'était le plan Fouchet, repris à deux avec l'Allemagne après son échec, dans le cadre du traité de l'Élysée. Appuyée sur cette Europe-là, Paris pourrait imposer une révision de l'Alliance atlantique et une réorganisation des rapports avec l'URSS permettant de dépasser, ou tout au moins de canaliser, la guerre froide et de rétablir un Concert européen, certes rénové, mais permettant de moins dépendre de la garantie américaine ${ }^{31}$.

Mais du point de vue de son organisation et de ses méthodes, la diplomatie française n'évoluait guère, en dehors d'une insistance encore plus forte sur la politique culturelle, marquée par le développement de la Direction générale des relations culturelles, scientifiques et techniques (DGRCST) au Quai d'Orsay ${ }^{32}$, et de l'apparition du concept nouveau de coopération, avec la création du ministère de la Coopération, chargé de se substituer aux anciennes relations coloniales ${ }^{33}$. Cependant, les avatars de cette nouvelle administration et les débats qui entouraient le concept même de coopération montrent que derrière le discours »tiers-mondiste« du général de Gaulle, qui paraissait annoncer la fin de la bipolarité et de certains aspects de la mondialisation, la politique française restait en fait une politique de grande puissance assez classique ${ }^{34}$. Un certain nombre de caractéristiques de cette politique le prouve: jusqu'en 1981, elle resta essentiellement centrée sur l'Afrique ex-française ou tout au plus francophone (Zaïre, Burundi, Rwanda). D'autre part, derrière une organisation complexe dominait en fait le rôle essentiel du président de la République: on était au cœur des enjeux de pouvoir de la $\mathrm{V}^{\mathrm{e}}$ République, et la coopération était avant tout un élément essentiel de la politique extérieure de la France: elle devait servir ses intérêts et son rayonnement. Le ministère de la Coopération, issu du secrétariat d'État aux relations avec la communauté, fut créé en mai 1961; il couvrait les anciennes colonies françaises en Afrique noire, plus les ex-colonies belges à partir de 196335. Mais dans une incontestable rivalité avec le Quai d'Orsay, malgré une tentative de répartition des tâches (les questions économiques, financières, culturelles, sociales, militaires, techniques pour la coopération, tandis que le Quai d'Orsay s'occupait de l'ensemble de l'Afrique, y compris des affaires politiques et de défense pour les pays ex-français ou belges), cela

31 ID., De Gaulle's France and the Soviet Union from Conflict to Détente, dans: Wilfried LOTH (dir.), Europe, Cold War and Coexistence, 1953-1965, Londres 2003, p. 173-189.

32 Albert SALON, L'Action culturelle de la France dans le monde, Paris 1983.

33 Pour l'organisation de l'instrument diplomatique français au début de la $\mathrm{V}^{\mathrm{e}}$ République, cf. Maurice VAÏSSE, La grandeur. Politique étrangère du général de Gaulle 1958-1959, Paris 1998.

34 À titre d'exemple, Georges-Henri Soutou, Le général de Gaulle et le Brésil (1958-1969), dans: Katia DE QueIros MatToso, Idelette MuZart-FonseCa dos Santos, Denis Rolland (dir.), Le Brésil, l'Europe et les équilibres internationaux, $\mathrm{XVI}^{\mathrm{e}}-\mathrm{XX}^{\mathrm{e}}$ siècles, Paris 1999, $\mathrm{p}$. 189-210.

35 Dmitri Georges LaVRoff (dir.), La politique africaine du général de Gaulle, Paris 1980. 
posait d'évidents problèmes de liaison et même d'orientation: le Quai d'Orsay prenant en compte la décolonisation plus franchement qu'une coopération que l'on aurait pu accuser à l'époque de »néo-colonialisme«. Finalement, le point de vue du ministère des Affaires étrangères, selon lequel il fallait reconnaître carrément l'indépendance des anciennes colonies et les traiter à l'instar des autres pays, et d'autre part que la politique française devait s'intéresser à l'ensemble de l'Afrique et pas seulement à l'ancienne Union française, triompha, au moins en apparence. À la suite du rapport de Jean-Marcel Jeanneney en 1963 sur la coopération (rapport très appuyé par la direction d'Afrique-Levant), le ministère de la Coopération devint, en 1966, un secrétariat d'État rattaché au Quai d'Orsay et, en 1969, celui-ci réorganisa la DGRCST: désormais en effet la coopération était conçue comme provisoire, devant cesser après la mise en place d'États viables. Mais d'autres organismes étaient également concernés: le ministère des Finances, le ministère de la Défense, le SDECE, le CEA, ou des sociétés comme ELF, acteur à part entière en Afrique ${ }^{36}$. La coordination de l'action de ces différents organismes, qui en fait le dessaisissaient largement, n'était pas assurée au niveau du Quai d'Orsay mais à celui de la présidence de la République, au secrétariat général auprès de la présidence pour la communauté et les affaires africaines et malgaches, dans une optique de politique très traditionnelle ${ }^{37}$.

Une certaine modernisation de l'action de la France commença avec Georges Pompidou (développement du thème de la francophonie, qui s'inscrit tout à fait dans la problématique de la mondialisation). Georges Pompidou fut sans doute le premier président français à réfléchir réellement à ce que nous appelons aujourd'hui la mondialisation (de Gaulle s'intéressait certes à l'ensemble du monde, mais dans un cadre conceptuel encore traditionnel). On retrouve la même dialectique de la pensée et de l'action au troisième niveau, le niveau international mondial. Notons d'abord que Georges Pompidou réfléchit profondément à la problématique du maintien de la diversité dans un monde en voie d'uniformisation. Comme il le déclara à l'UNESCO le 17 mars 1970, il était convaincu que les civilisations »s'enrichissaient réciproquement par leur interpénétration« mais qu'il fallait lutter »contre la tendance à une uniformisation qui serait un appauvrissement collectif« ${ }^{38}$. Et, joignant l'action à la pensée, il était convaincu que cette diversité des civilisations procurait à la France un champ d'action pour lutter contre les blocs auxquels il était aussi opposé que le Général. L'instrument privilégié dans ce domaine serait la francophonie. Dans sa très intéressante étude »La francophonie et les relations extérieures«, Laurence Saint-Gilles a très bien montré comment la francophonie se situait au cœur de la politique internationale de Georges Pompidou au niveau mondial, ainsi qu'au croisement de ses conceptions culturelles et

36 Soutou, Pierre Guillaumat (voir n. 3).

37 On peut s'en convaincre facilement en étudiant le journal du responsable des questions africaines à l'Élysée, Jacques Foccart, Journal de l'Élysée, Tomes I-III, Pars 1997-1999.

38 Georges POMPIDOU, Entretiens et discours, Paris 1984, p. 179. 
politiques ${ }^{39}$. En effet, la langue française était au centre de l'universalisme du message français mais était en même temps, selon Georges Pompidou, »un des moyens qu'avait l'Europe occidentale d'affirmer sa personnalité vis-à-vis des États-Unis«. Le 19 février 1971, il assigna au Haut Comité pour la défense et l'expansion de la langue française, qu'il avait suscité en 1966, trois objectifs: faire des 150 millions de francophones putatifs répartis dans le monde de vrais francophones, faire du français la langue de communication de la Communauté européenne, assurer sa présence sur les cinq continents. Vers l'Afrique noire, vers l'Europe latine, vers l'Allemagne mais aussi partout dans le monde, la défense de la langue française devrait illustrer la dialectique essentiellement civilisatrice entre le particulier et l'universel et en même temps contribuer à éviter l'uniformisation culturelle, autre façon aussi de lutter contre les blocs tout en développant l'influence française dans un domaine qui ne pouvait pas donner prise au soupçon d'impérialisme ou de néo-colonialisme. Le dialogue des civilisations supposait en effet la reconnaissance et la transmission de valeurs universelles et était pour Georges Pompidou la vraie réponse à la globalisation.

À l'extrême fin de la présidence de Georges Pompidou, en 1974, Michel Jobert, alors ministre des Affaires étrangères, créa le Centre d'analyse et de prévision. Le CAP joua à partir de là un rôle essentiel dans la réflexion du Quai d'Orsay, en particulier sur des problèmes de fond dépassant l'horizon quotidien des directions et en établissant le lien avec la recherche universitaire. Les thèmes abordés, y compris les rapports transatlantiques, le problème soviétique, les questions de sécurité et de désarmement, permettaient de suivre l'évolution du monde du point de vue des problèmes soulevés ici. D'autre part, le passage de certains diplomates au CAP permettait ensuite d'irriguer les directions du résultat de leurs réflexions ${ }^{40}$. Cela dit, il ne faut pas croire que le CAP poussait particulièrement vers une européanisation de la politique extérieure française; au contraire, le thème du passage d'un monde »bipolaire « à un monde »multipolaire«, qui naquit dans le cadre de ce centre, permettait de montrer que la France pouvait retrouver une nouvelle liberté d'action à partir de l'érosion des blocs.

Valéry Giscard d'Estaing poursuivit l'adaptation progressive de la politique française à l'évolution du monde occidental et de l'Europe en suscitant la création du G7, celle du Conseil européen des chefs d'État et de gouvernement et l'élection du Parlement européen au suffrage universel ${ }^{41}$. Il soutint les efforts de certains membres du Quai d'Orsay pour introduire dans le programme de la CSCE d'Helsinki la »troisième corbeille«, celle des droits de l'homme ${ }^{42}$. Les diplomates s'adaptaient au système de

39 Laurence SAINT-GiLles, La francophonie et les relations extérieures, dans: Jean-Claude Groshens, Jean-François SiRINELli (dir.), Culture et action chez Georges Pompidou, Paris, p. 373-393.

40 Le débat de politique étrangère française 1974-2004. Centre d'anlyse et de prévision, $30^{\mathrm{e}}$ anniversaire, éd. par le ministère des Affaires étrangères (2005). On trouvera certaines études du CAP en ligne sur le portail du Quai d'Orsay.

41 Serge BERSTEIN, Jean-François SiRINELLi (dir.), Les années Giscard. Valéry Giscard d'Estaing et l'Europe 1974-1981, Paris 2006.

42 Jacques ANDRÉANI, Le Piège. Helsinki et la chute du communisme, Paris 2005. 
diplomatie globale du G7, prenaient de plus en plus l'habitude de la coopération politique introduite entre les ministères des Affaires étrangères à partir de 1972 et collaboraient de façon de plus en plus intime en particulier avec l'Auswärtigen Amt allemand. D'autre part, l'importance à nouveau cruciale des questions stratégiques et du désarmement relançait l'importance du Service des pactes et des affaires stratégiques, tandis que des membres du Quai d'Orsay devenaient des spécialistes internationalement reconnus des questions stratégiques ${ }^{43}$. En 1976-1978 une réforme du Quai d'Orsay, la première depuis 1945 et connue sous le nom de "géographisation«, distingua clairement entre les affaires bilatérales d'une part (du ressort des directions géographiques) et les affaires multilatérales, apanage de la Direction politique et de la Direction économique. L'importance croissante des affaires multilatérales dans un monde de plus en plus complexe et interdépendant était ainsi prise en compte. Cependant, les chocs pétroliers réactivaient une tradition de »libéralisme organisé«, réponse française depuis 1914 à la mondialisation libérale d'inspiration anglo-saxonne et parfaitement adaptée à la formation économique étatiste reçue par les énarques ${ }^{44}$.

François Mitterrand devait en fait poursuivre ces différentes orientations, la seule nouveauté réelle résidant dans la relance de la coopération, dont le ministère, avec Jean-Pierre Cot, ne limitait plus son action à l'ancien pré carré français mais l'étendait à l'ensemble du monde en voie de développement. Mais, malgré sur ce point la claire prise en compte de la mondialisation, qui correspondait aussi au fait que l'on souhaitait riposter au défi de la mondialisation libérale impulsée par les États-Unis, la politique extérieure et les réflexes des diplomates ne changeaient pas en profondeur: c'était toujours l'exploitation au profit des objectifs français des équilibres croisés déjà cités. La politique française préservait en fait son identité grâce aux circonstances exceptionnelles induites par la guerre froide qui maintenait l'Allemagne divisée, évitait à la France, pour la première fois dans son histoire, de se retrouver en première ligne dans les tensions européennes, et qui valorisait son rôle international, tout en lui assurant automatiquement - et quelles que fussent les manifestations de son indépendance - la garantie américaine ${ }^{45}$. Ce confort intellectuel explique sans doute que la diplomatie française n'ait guère vu venir l'automne de 1989, et n'ait admis la réalité inéluctable d'une réunification allemande pure et simple qu'après les élections en RDA de mars

43 Outre Jean-Louis Gergorin, deuxième directeur du CAP après Thierry de Montbrial, citons François de Rose (qui fut représentant auprès de l'OTAN): cf. par exemple François DE ROSE, La France et la défense de l'Europe, Paris 1976.

44 Georges-Henri Soutou, Henri Hauser et la Première Guerre mondiale, dans: SéverineAntigone MARIN, Georges-Henri SOUTOU (dir), Henri Hauser (1866-1946). Humaniste, Historien, Républicain, Paris 2006, p. 147-184. Rappelons que Pierre Mendès France s'engagea fortement à partir des années 1960 pour le contrôle et la régulation au niveau international du prix des matières premières, en particulier sur les conseils du diplomate Stéphane Hessel (ID., Danse avec le siècle, Paris 1997) et ses nombreuses publications et rapports sur l'aide au tiers monde).

45 On lira avec intérêt le point de vue de François PUAUX, ancien directeur politique, qui a tenu après son départ à la retraite la rubrique de politique étrangère de la Revue des deux mondes: ID., La politique internationale des années quatre-vingt, Paris 1989. 
$1990^{46}$. Que la position de la majorité des diplomates avait été sur ce point très proche de celle du Président nous est confirmé par François Scheer, alors secrétaire général du Quai ${ }^{47}$.

\section{0-2006 UNE MODERNISATION TARDIVE ET LIMITÉE?}

Il fallut attendre la fin de la guerre froide, le traité de Maastricht, la création de la PESC et l'accélération évidente de la mondialisation, pour que dans les années 1990 le Quai d'Orsay se réorganise profondément, même si le réseau des ambassades ne fut pas réformé à l'instar de l'administration centrale. Une première réforme eut lieu en 1993, corrigée d'ailleurs en 1998. Désormais, la nouvelle Direction générale des affaires politiques et de sécurité, dont le responsable est également secrétaire général adjoint, prend pleinement en compte la mondialisation des questions politiques (lui sont subordonnées la Direction des Nations-Unies et des organisations internationales, la Direction des affaires stratégiques, de sécurité et de désarmement, la Direction de la coopération militaire et de défense, la mission de liaison avec les organisations internationales non gouvernementales) et la montée de la personnalité internationale de l'Union européenne (avec le Service de la politique étrangère et de sécurité commune). On notera que l'Alliance atlantique et les rapports avec les États-Unis ne sont pas au premier plan de cet organigramme (auparavant le Service des pactes, dont c'était en fait le domaine, relevait directement du secrétariat général).

La direction d'Europe traditionnelle a été transformée: on a désormais une Direction de la coopération européenne pour l'Europe communautaire, à l'organisation clairement liée au multilatéralisme dans le cadre européen, et une Direction de l'Europe continentale pour l'Europe non communautaire, tandis que pour le reste du monde subsistent les directions géographiques traditionnelles. Désormais, les deux principales

46 La politique extérieure de François Mitterrand fait toujours l'objet de vifs débâts. Le meilleur exposé de l'un de ses acteurs essentiels: Hubert VéDRINE, Les mondes de François Mitterrand, Paris 1996. Certains diplomates la critiquèrent fermement: Jacques JESSEL, La double défaite de Mitterrand, Paris 1992. D'autres, d'obédience gaulliste, se montrèrent plus sensibles au fait que certains de ses axes n'étaient pas, au fond, incompatibles avec les orientations précédentes de la $V^{e}$ République (Jean-Bernard RAIMOND, Le Quai d'Orsay à l'épreuve de la cohabitation, Paris 1989). Nous commençons à avoir des travaux scientifiques, qui tendent à réviser de façon positive l'interprétation de la politique de François Mitterrand: Tilo SCHABERT, Wie Weltgeschichte gemacht wird. Frankreich und die deutsche Einheit, Stuttgart 2002, et Frédéric Bozo, Mitterrand, la fin de la guerre froide et l'unification allemande, Paris 2005. Mais je maintiens intégralement le point de vue présenté ici, développé plus à fond dans: L'Alliance incertaine (voir n. 14). L'ouvrage édité par Samy COHEN, Mitterrand et la sortie de la guerre froide, Paris 1998 , reste indispensable et présente tous les points du débat.

47 Dans: COHEN, Mitterrand (voir n. 46), p. 53. Cf. également la contribution de Jacques BARIÉTY, François Mitterrand, Willy Brandt et la réunification de l'Allemagne, dans: Horst MöLLER, Maurice VAÏSSE (dir.), Willy Brandt und Frankreich, Munich 2005, p. 247-256. 
directions (Direction générale des affaires politiques et de sécurité et Direction de la coopération européenne) prennent de toute évidence directement en compte mondialisation et européanisation, pendant que la Direction des affaires économiques et financières réformée s'inscrit clairement dans le cadre de la mondialisation (en particulier avec sa sous-direction de l'environnement et celle de l'énergie, des transports et des nouvelles technologies). Quant à la Direction générale de la coopération internationale et du développement (DGCID qui descend de la DGRST), elle s'inscrit pleinement dans la mondialisation, par exemple avec sa Cellule de mobilisation sur les projets multilatéraux. La diplomatie multilatérale, dans une perspective mondiale et européenne, est passée désormais au centre de l'action du Quai d'Orsay.

Dans cette organisation rénovée, un axe essentiel est une collaboration étroite avec Berlin en particulier, au niveau des services des deux ministères. Cette collaboration est l'une des bases de la PESC depuis le traité de Maastricht.

D'autre part, certains diplomates, sur le plan intellectuel, participent pleinement aux débats actuels sur ces sujets, y compris les débats théoriques sur la mondialisation et le »postnational«. Citons »L'ordre mondial«, manuel classique publié par Philippe Moreau Defarges, diplomate détaché à l'IFRI, ou encore »L'avenir de la liberté: la démocratie et la mondialisation«, publié en 1999 par Jean-Marie Guéhenno, ancien directeur du Centre d'analyse et de prévision du Quai d'Orsay. Citons également Thierry de Montbrial, actuel directeur de l'IFRI et premier directeur du CAP: »L'action et le système du monde«, Paris 2002.

Il existe cependant d'autres courants: des ministres comme Hubert Védrine et Dominique de Villepin se sont abondamment exprimés et ont publié sur l'évolution actuelle du système internationale après la fin de la guerre froide et à l'heure de l'»hyperpuissance américaine « ${ }^{48}$. Néanmoins, une comparaison des écrits de ces deux ministres avec le livre de leur collègue allemand Joschka Fischer montre bien que beaucoup de responsables français restent en retrait par rapport à l'européanisation et à la mondialisation, sans parler de l'atlantisme récusé depuis les années $1950^{49}$. Quant aux diplomates, on trouve une large gamme d'opinions sur ces sujets.

En effet, l'accent mis régulièrement sur la »multipolarité« au plan international et l'évocation d'un monde reposant sur le jeu d'un ensemble de grandes puissances (Union européenne, États-Unis, Russie, Chine, Inde...) correspond en fait (outre bien sûr l'opposition sous-jacente aux États-Unis) à une vision encore assez classique du système international ${ }^{50}$. Les projets récurrents de »coopérations renforcées« ou de

48 Hubert VÉDRINE, Face à l'hyperpuissance: textes et discours 1995-2003, Paris 2003; Dominique DE VILLEPIN, Un autre monde, Paris 2003, ainsi que son interview par Politique internationale $102(2003 / 2004)$, supplément.

49 Joschka FISCHER, Die Rückkehr der Geschichte. Die Welt nach dem 11. September und die Erneuerung des Westens (Le retour de l'histoire. Le monde après le 11 septembre et la rénovation de l'Occident, Cologne 2005.

${ }_{50}$ Cf. comme exemple de l'école réaliste rénovée, le livre de Thierry DE MONTBRIAL, L'action et le système du monde, cité ci-dessus (compte rendu de Georges-Henri SOUTOU dans: Revue d'histoire diplomatique [2002]/4, p. 375-380). 
"groupes pionniers" dans le cadre de l'Union européenne sont aussi une façon d'écarter le fédéralisme européen que préconise Berlin, au profit d'une vision qui reste davantage interétatique. La propension à encourager des groupes ad hoc, comme le groupe de contact pour l'ex-Yougoslavie, vise sans doute surtout à maintenir tant bien que mal un rôle international de puissance nucléaire à responsabilités mondiales, rôle conçu de façon toujours assez classique dans un environnement européen, atlantique et mondial pourtant bouleversé51. Et l'idée parfois évoquée de transférer les sièges qu'occupent au Conseil de sécurité de l'ONU la France et la Grande-Bretagne à l'Union européenne ne suscite aucun enthousiasme évident: Paris préfère défendre l'idée d'un élargissement du cercle des membres permanents du Conseil, en particulier à l'Allemagne. Lors du colloque anniversaire du CAP en 2004, Philip Gordon pouvait souligner le conservatisme foncier de la diplomatie française, cherchant à maintenir un rôle mondial en s'appuyant sur une »Europe-puissance« dont le génome, pour ainsi dire, ne s'écartait guère de celui du plan Fouchet ${ }^{52}$.

Cela dit, les choses évoluent: par exemple la diplomatie française traite maintenant du conflit israélo-palestinien largement dans le cadre du »quartette (États-Unis, Russie, ONU, UE) et en concertation croissante avec l'Union européenne. La diplomatie française est de façon générale très active dans la gestion des crises, là aussi dans le cadre, complexe et qui échappe largement à l'opinion publique, du processus de décision de l'UE en matière internationale ${ }^{53}$. D'autre part, on pourrait remarquer que la France n'est pas la seule à maintenir une diplomatie de type classique: après tout, l'affaire du nucléaire iranien est traitée conjointement par la Grande-Bretagne, l'Allemagne et la France, dans un esprit qui n'est pas si éloigné que cela du Concert européen traditionnel. En fait, les réalités interétatiques et la nécessité d'une diplomatie traditionnelle restent incontournables. Les récents conflits, y compris ceux des Balkans, ont montré les limites des thèses "postnationales«, car ce sont finalement des États qui sont intervenus pour les stopper. Mais ils ont montré aussi les limites du droit d'ingérence, dans la mesure où pour le moment la Bosnie et le Kosovo restent des protectorats internationaux, sans que des solutions définitives et stables paraissent à portée de main. Les incertitudes sur l'avenir du système international ne peuvent que se traduire dans l'organisation du travail des diplomates et la conception qu'ils se font de leur rôle.

La conclusion ne peut être que prudente et provisoire. On est bien conscient que la conception de l'identité nationale de type traditionnel, sur le plan de la diplomatie et de la politique extérieure, doit évoluer. On résiste par exemple à l'américanisation sur tous les plans, y compris à l'OTAN, à l'ONU, et par l'»exception culturelle«, mais on s'efforce de resituer ce combat dans un contexte global, dans une vision du monde qui

51 Sur le rôle des diplomates français dans ce groupe de contact, cf. Francine BoIDEVAIX, L'Europe gère les Balkans. La responsabilité finale reste au Concert des puissances, dans: Relations internationales 121 (2005), p. 91-108.

52 Cf. Le débat de politique étrangère française 1974-2004 (voir n. 40), p. 94-100.

53 Agnieszka NowAK (dir.), Civilian crisis management: the EU way, Paris 2006 (Chaillot Paper 90, Institut d'études de sécurité de l'Union européenne). 
se veut universelle et dépassant le seul intérêt français. Sur ce point, le langage a subtilement évolué depuis les années 1960.

D'autre part, si on compare les conceptions et l'activité des diplomates français et l'organisation du Quai d'Orsay aujourd'hui à ce qu'ils étaient en 1945 ou au début des années 1950, on constate que la diplomatie bilatérale a beaucoup perdu de son importance par rapport à la diplomatie multilatérale (qui avait assez mauvaise presse en 1945 à la suite de l'échec de la sécurité collective d'avant-guerre) et que la coopération européenne en liaison avec Bruxelles (et Berlin) a pris une place majeure.

Mais les conséquences de la mondialisation libérale sont toujours seulement partiellement prises en compte (comme le montrent les réticences de la diplomatie française à envisager une transformation de la politique agricole commune et les appels répétés des gouvernements successifs aux diplomates pour qu'ils se mettent davantage au service des intérêts économiques nationaux). Et l'européanisation n'est acceptée que dans certaines limites, qui ne vont pas au-delà de la mise en place d'une étroite concertation multilatérale: l'évocation de l'»Europe-puissance«, liée au concept de »groupes pionniers «, servant peut-être surtout à tenter de recycler un modèle français finalement encore assez traditionnel. L'avenir dira si celui-ci, après l'échec du traité constitutionnel européen, l'enlisement de la politique américaine et les difficultés d'une modernisation encore fort peu maîtrisée, reste finalement pertinent, ou si les diplomates français et leurs dirigeants devront accepter des évolutions encore plus radicales vers une véritable diplomatie européenne et un bouleversement de la diplomatie interétatique traditionnelle.

\section{DEUTSCHE ZUSAMMENFASSUNG}

Nationale Identität, Amerikanisierung, Europäisierung, Globalisierung: Diese Problematik steht natürlich im Zentrum der französischen Diplomatie seit 1945. Selbstverständlich ist diese durch Weiterentwicklungen und Anpassungen geprägt, aber auch durch gewisse Kontinuitäten, insbesondere die Sorge, die nationale Identität zu bewahren: Frankreich ist immer das am wenigsten atlantische unter den westeuropäischen Ländern gewesen und das am meisten widerspenstige gegenüber der amerikanischen Führung, und seine Eliten haben im allgemeinen der liberalen Globalisierung mißtraut. Was die Europäisierung anbetrifft, so hat sie sicherlich eine Achse der französischen Diplomatie und der französischen Diplomaten dargestellt, aber sie ist Stückwerk geblieben und belastet durch sehr »nationale« Hintergedanken. Selbst heute noch werden die Konsequenzen der liberalen Globalisierung immer nur teilweise in Rechnung gestellt. Und die Europäisierung ist nur in gewissen Grenzen anerkannt, die nicht über die Herstellung einer engen multilateralen Abstimmung hinausgehen: Das Wachrufen einer »Europa-Macht«, verbunden mit dem Konzept von »Pioniergruppen«, dient vielleicht vor allem dazu zu versuchen, ein französisches Modell wiederzuverwerten, das letztlich noch ziemlich traditionell ist. Die Zukunft wird zeigen, ob es am Ende, nach dem Mißerfolg des europäischen Verfassungsvertrags, dem Erlahmen der amerikanischen Politik und den Schwierigkeiten einer noch sehr wenig gemeisterten Modernisierung, richtig bleibt oder ob die französischen Diplomaten und ihre Führungsspitzen noch viel radikalere Fortentwicklungen akzeptieren müssen hin zu einer europäischen Diplomatie und einem Umsturz der traditionellen zwischenstaatlichen Diplomatie. 